\title{
El cuidado de enfermería en pacientes con riesgo cardiovascular sustentado en la teoría de Nola J. Pender ${ }^{1}$
}

\author{
Julio César Cadena Estrada² \\ Yariela González Ortega³
}

doi:10.11144/Javeriana.ie19-1.ecep

Cómo citar: Cadena Estrada JC, Gonzáles Ortega Y. El cuidado de enfermería en pacientes con riesgo cardiovascular sustentado en la teoría de Nola J Pender. Investig Enferm. Imagen Desarr. 2017;19(1): 107-121. http://dx.doi.org/10.11144/Javeriana.ie19-1.ecep

1. Artículo de reflexión. Fecha de recepción: 15 de Enero de 2015. Fecha de aceptación: 15 de Enero de 2016.

2. Maestrando en Enfermería con orientación en Administración de la Universidad Nacional Autónoma de México. Departamento de Investigación en Enfermería del Instituto Nacional de Cardiología Ignacio Chávez. Correo electrónico: cadjul@cardiologia.org.mx

3. Doctora en Enfermería. Docente de la Facultad de Enfermería, Universidad de Panamá. Correo electrónico: yarielag11@gmail.com 


\section{Resumen}

Introducción: Las enfermedades crónico-degenerativas como la Enfermedad Coronaria, tienen su origen en diversos factores de riesgo, hábitos y estilos de vida no saludables, por lo que la prevención y promoción de la salud basada en teorías o modelos de enfermería, cumplen un papel fundamental en la solución de los problemas de salud pública. Objetivo: Elaborar una propuesta de aplicación de cuidado de enfermería a los pacientes con riesgo cardiovascular sustentado en el Modelo de Promoción de la Salud (MPS) de Nola J Pender. Propuesta: Se plantea el cuidado de las personas con base en el MPS donde el profesional de enfermería: 1) determine basalmente la autoeficacia percibida, la percepción de su salud, los factores de riesgo, el perfil de estilos de vida saludable y los factores personales; 2) intervenga con un curso de educación para con la participación de un "paciente modelo" (3-12 sesiones de 30-60 minutos durante la(s) semana(s) de hospitalización) y seguimiento domiciliario con talleres y herramientas escritas; 3) evaluar el efecto de la intervención; y 4) dar seguimiento con las tecnologías de la información y comunicación Conclusión: El cuidado de enfermería del paciente con riesgo cardiovascular basado en el MPS, utilizando tecnologías de la información y comunicación, puede influir en los factores cognitivos-perceptuales, las barreras de acción, los beneficios percibidos, los afectos, la competencia y sobre todo en la autoeficacia percibida, modificando de forma activa, vicaria, persuasiva o afectiva su conducta de riesgo. No obstante, es fundamental considerar los factores personales, interpersonales, situacionales y la conducta previa en las intervenciones educativas de enfermería.

Palabras clave: atención de enfermería; autoeficacia; factores de riesgo; enfermedades cardiovasculares; enfermería cardiovascular; tecnología de la información

\section{Nursing care in patients with cardiovascular risk based on the theory of Nola J. Pender}

\section{Abstract}

Introduction: chronic degenerative diseases such as coronary heart disease are caused by various risk factors, habits and styles of unhealthy life, so that prevention and health promotion based on theories or models of nursing, play an essential role in solving public health problems. Objective: To develop a proposal for implementation of nursing care to patients with cardiovascular risk supported by the Health Promotion Model (HPM) of Nola J Pender. Proposal: The care of people is proposed based on the HPM where the nurse: 1)basally determines the self-efficacy perceived, health perception, risk factors, the profile of healthy lifestyles and personal factors; 2) gets involved in an education course with the participation of a "model patient" (3-12 sessions of 30-60 minutes during the (s) week (s) of hospitalization) and home monitoring with workshops and written tools; 3) evaluates the effect of the intervention; and 4) follows up with information technology and communication. Conclusion: Nursing care of patients with cardiovascular risk based on the HPM, using information technology and communication, can influence cognitive-perceptual factors, barriers to action, perceived benefits, affections, competition and especially the perceived self-efficacy, modifying active, vicarious, persuasive or emotional risk behavior. However, it is essential to consider the personal, interpersonal, situational factors and prior conduct in educational nursing interventions.

Keywords: nursing care; self-efficacy; risk factors; cardiovascular diseases; cardiovascular nursing; Information Technology 


\section{O cuidado de enfermagem em pacientes com risco cardiovascular baseado na teoria de Nola J. Pender}

\section{Resumo}

Introdução: As doenças crônico-degenerativas como a Doença Coronária tem origem em diversos fatores de risco, hábitos e estilos de vida não saudáveis, pelo que a prevenção e promoção da saúde baseada em teorias ou modelos de enfermagem, desempenham papel basilar na resolução de problemas de saúde pública. Objetivo: Elaborar proposta de aplicação de cuidado de enfermagem a pacientes com risco cardiovascular sustentado no Modelo de Promoção da Saúde (MPS) de Nola J Pender. Proposta: Colocar-se o cuidado das pessoas com base no MPS onde o profissional de enfermagem: 1) vai determina em jejum a auto-eficácia percebida, a percepção da sua saúde, os fatores de risco, o perfil de estilos de vida saudáveis e os fatores pessoais; 2) intervir com curso de ensino com a participação de um "paciente modelo" (3-12 sessões de 30-60 minutos durante a(s) semana(s) de hospitalização) e monitoramento domiciliar com oficinas e ferramentas escritas; 3) avaliar o efeito da intervenção; e 4) fazer seguimento com as tecnologias de informação e comunicação. Conclusão: O cuidado de enfermagem do paciente com risco cardiovascular baseado no MPS, utilizando tecnologias de informação e comunicação, pode influenciar nos fatores cognitivos-perceptuais, as barreiras de ação, os benefícios percebidos, os afeitos, a concorrência e por sobre todo na auto-eficácia percebida, alterando de forma ativa, vicária, persuasiva ou afetiva sua conduta de risco. No entanto, é fundamental considerar os fatores pessoais, interpessoais, situacionais e a conduta prévia nas intervenções educacionais de enfermagem.

Palavras-chave: atendimento de enfermagem; auto-eficácia; fatores de risco; doenças cardiovasculares; enfermagem cardiovascular; tecnologia de informação. 


\section{Introducción}

Las teorías y modelos de enfermería son una plataforma teórica para el proceso de cuidado de la persona sana o enferma, sí bien no son la verdad absoluta, si permiten guiar al profesional de enfermería en la solución de problemas de salud.

El Modelo de Promoción de la Salud (MPS) de Nola J. Pender pretende desde sus principales supuestos explicar la relación de los factores que influyen en las conductas de salud. Este artículo tiene la finalidad de generar una propuesta de cuidado de enfermeria al paciente con riesgo cardiovascular relacionando con los principales supuestos del MPS y el uso de las Tecnologías de la Información y Comunicación en salud (TIC's).

Es así como el trabajo de enfermería es fundamental dentro de la prevención y promoción de la salud, ya que puede incidir en la modificación de conductas no saludables por conductas promotoras de salud, y por lo tanto, el profesional de enfermería podrá colaborar en la disminución de la recidiva de la Enfermedad Coronaria (EC), el número de reingresos hospitalarios, mejorar la calidad de vida del paciente a largo plazo y favorecer una rápida reincorporación al área laboral y social de la persona cardiópata.

\section{Desarrollo del tema}

De acuerdo a la Organización Mundial de la Salud (OMS), las enfermedades crónico-degenerativas representan una de las principales causas de muerte en países desarrollados y emergentes (1). En México estos problemas de salud están ligados a factores de riesgo acumulativos y sinérgicos que propician una mayor incidencia de enfermedades cardiovasculares, que afectan tanto a hombres como mujeres.

Los factores de riesgo son características o conductas de las personas que aumentan la probabilidad de sufrir una enfermedad. La combinación de una dieta poco saludable, la falta de actividad física o sedentarismo, el consumo de tabaco, la ingesta excesiva de alcohol, entre otros; son el origen de padecimientos como la hipertensión arterial sistémica, la obesidad, la hipercolesterolemia, la hipertrigliceridemia, hiperglucemia, diabetes mellitus tipo 2, condiciones que a su vez son Factores de Riesgo Cardiovascular (FRCV).

Desde este punto de vista, cobra gran importancia la prevención y promoción de la salud a través de la modificación de hábitos y estilos de vida poco saludables y el control de los FRCV para disminuir el riesgo, la recidiva y la progresión de la Enfermedad Coronaria (EC). Para la modificación de los factores de riesgo, el MPS de Nola J Pender (fig. 1) identifica en el individuo factores cognitivo-perceptuales que son modificados por las características situacionales, personales e interpersonales, promoviendo la realización de conductas favorecedoras de salud, guiadas por pautas para la acción (2). 


\section{CARACTERÍSTICAS Y EXPERIENCIAS INDIVIDUALES}

\section{CONOCIMIENTOS Y AFECTOS ESPECÍFICOS DE LA CONDUCTA}

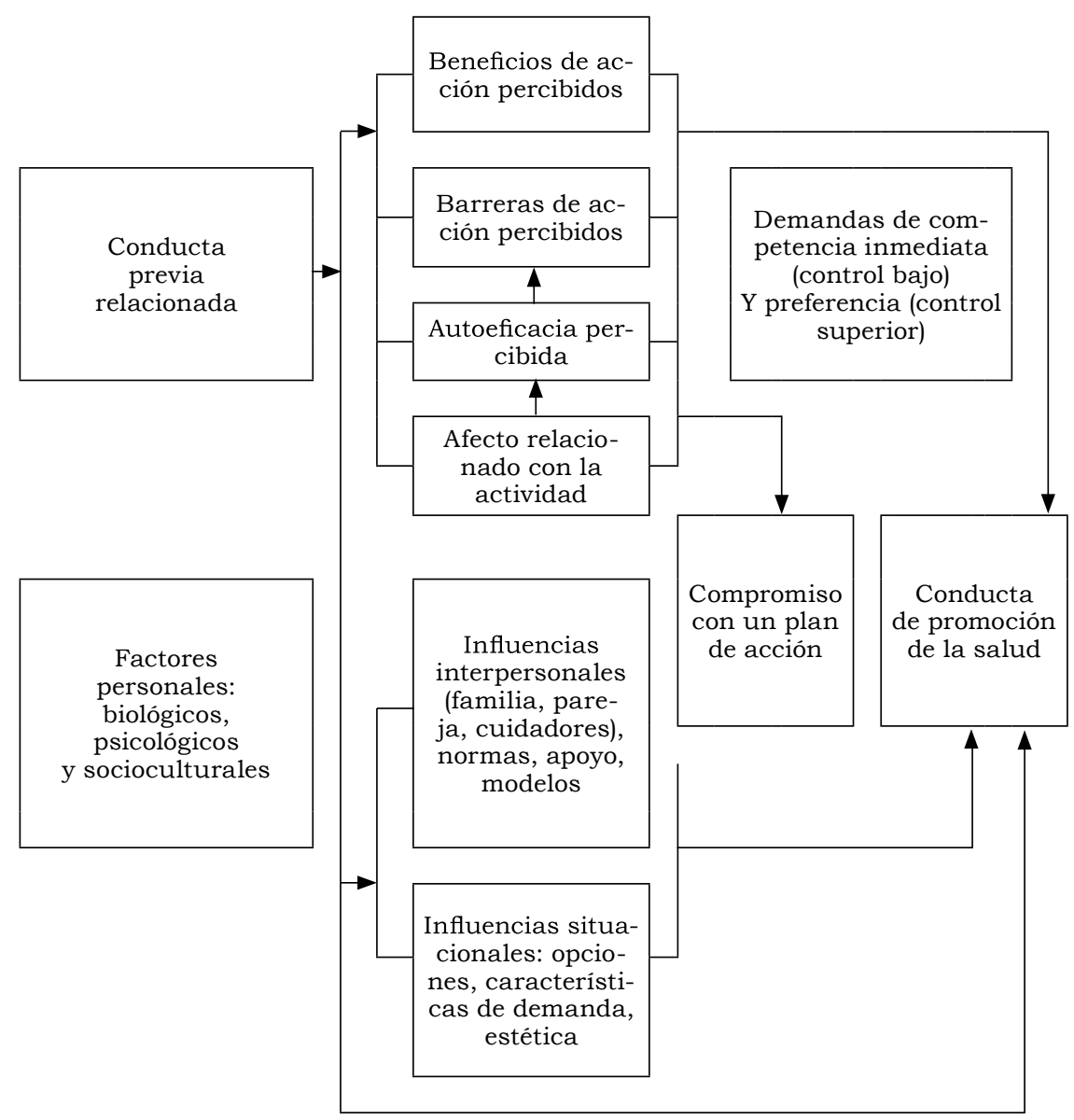

Figura 1. Modelo de Promoción de la Salud de Nola J. Pender

Fuente: Marriner TA, Raile AM. Modelos y teorias de enfermería, 2007 (2).

Pender, basada en Bandura y Feather, considera que los aspectos del aprendizaje cognitivo y conductual son factores psicológicos que influyen en los comportamientos de las personas. Señala cuatro requisitos para modelar el comportamiento: atención (estar expectante ante lo que sucede), retención (recordar lo que uno ha observado), reproducción (habilidad de reproducir la conducta) y motivación (una buena razón para querer adoptar esa conducta) $(2,4)$. Así mismo, el componente motivacional clave para conseguir un logro, es la intencionalidad, entendida como el compromiso personal con la acción. Este aspecto es representativo en el análisis de los comportamientos voluntarios, 
dirigidos al logro de las metas propuestas (5). En la vida diaria, los seres humanos observan lo que hacen otras personas. Desde la niñez se aprende por imitación, lo que hacen los padres o modelos a seguir influye en las conductas, y por lo tanto en la salud de sus predecesores. Si éstos consumen una dieta rica en grasa, carbohidratos, altas cantidades de sal, toman gaseosa en lugar de agua, se divierten en actividades que requieren poca actividad física como los video-juegos o pasan periodos prolongados frente a la televisión, estos comportamientos serán imitados por la siguiente generación, desde la juventud, traduciéndose en el padecimiento de enfermedades cardiovasculares en años posteriores. También, es necesario considerar los factores personales (biológicos, psicológicos y socioculturales) ya que son predictivos de conductas saludables y no saludables; por lo tanto, influyen en las metas planteadas (6-15).

Actualmente las intervenciones de enfermería que se llevan a cabo en las instituciones de salud están relacionadas con la modificación de los FRCV, y por ende, de los hábitos y estilos de vida poco saludables. A través de educación para la salud, con técnicas participativas se conduce a la toma de conciencia tratando de empoderar al individuo para que de forma autónoma analice y cambie sus comportamientos y actitudes (15). A través del MPS, el cuidado que proporciona enfermería pretende promover que los pacientes busquen regular de forma activa su propia conducta; es decir, que identifiquen en sí mismos FRCV, o bien EC, La enfermera incentiva al individuo a modificar su conducta previa, adquiriendo nuevos hábitos saludables. Así mismo, se motiva a las personas para que transformen progresivamente su entorno teniendo en cuenta la complejidad biopsicosocial, esto es importante debido a que es necesario considerar el contexto para modificar estilos y hábitos de vida no saludables.

La enfermera desde el concepto de autoeficacia propuesto por Pender debe lograr una modificación de las conductas de riesgo y promocionar aquellas que benefician a las personas. Se ha observado que los cambios de conducta se realizan en forma activa cuando los profesionales de enfermería motivan en las personas su capacidad de iniciarlos; sin embargo, es necesario considerar que la autoeficacia percibida, término también utilizado por Pender, influye sobre las barreras de acción percibida, de manera que la mayor eficacia, conlleva una menor percepción de las barreras para el desempeño de esta conducta (16). En la medida que el individuo se siente motivado, percibe que tiene la capacidad de hacer cambios en su estilo de vida y observa los beneficios a corto plazo de hábitos como ingerir una dieta sana, equilibrada y balanceada; hacer ejercicio físico de moderado a intenso o por lo menos caminar; evitar el consumo de tabaco y alcohol; entre otros. Por consiguiente, las personas se comprometen a adoptar estas conductas saludables (17-21). Los profesionales de enfermería, desde la promoción de la salud, deben considerar que sus intervenciones educativas logren conductas positivas, más por gratificación, que por temor. Cuanto mayor sea el compromiso con un plan específico de acción, más probable es que se mantengan las conductas promotoras de salud a lo largo del tiempo. Por esto, la enfermera debe considerar un plan de intervención que se refuerce durante un periodo considerable de tiempo, o por lo menos con un año de seguimiento. 
Ahora bien, según Pender, la competencia percibida de la eficacia de uno mismo para ejecutar una cierta conducta, aumenta la probabilidad de que las personas se comprometan con la acción. La enfermera debe considerar diversos factores para que la persona con FRCV o EC modifique sus hábitos y estilos de vida no saludable; entre los cuales destacan: a) la conducta previa y las características heredadas y adquiridas; b) el modelamiento, donde es más probable que las personas se comprometan a adoptar conductas de promoción a la salud cuando los individuos importantes para él/ella modelan su conducta, ofrecen ayuda y apoyo para permitirla; y c) la modificación de los conocimientos, el afecto y el entorno interpersonal y físico $(3,22)$. En la práctica clínica, las intervenciones educativas implementadas a través de material audiovisual para controlar la hipertensión arterial, la diabetes mellitus tipo 2, disminuir la hipercolesterolemia y aumentar la actividad física, entre otros; deben trazar metas cortas y reales. Esta estrategia puede apoyarse en un personaje o modelo social que influya en las creencias previas, el afecto y la promulgación de las conductas de promoción a la salud, para asegurar que la intervención de enfermería tenga más probabilidad de éxito.

En países como México, es imprescindible que el cuidado de enfermería sea cada día más visible al dar solución a problemas de salud de la población; y es a través de los principales postulados de Pender que esto se puede conseguir, ya que el MPS ha demostrado ser aplicable en la práctica clinica (23).

Se han realizado diversos estudios en países como México (25), Colombia (26 -28), Brasil (29-32), Corea del sur (33-35), Estados Unidos (36-40), Iran (41), entre otros; enfocados en grupos poblacionales que consideran desde niños (6 años) hasta adultos mayores (>65 años) con enfermedades crónicas (hipertensión arterial sistémica, diabetes mellitus, artritis, osteoporosis, cardiopatías congénitas), factores de riesgo (obesidad, sedentarismo o inactividad física) y estilos de vida no saludables. Se ha comprobado que la percepción del estado de salud, la autoeficacia, la educación formal, la autonomía, los conocimientos previos, los factores ambientales, socioculturales y psicológicos, el tipo de relación familiar en la vivienda y la situación o percepción de salud, son los principales predictores de conductas promotoras de salud que motivan a realizar cambios en los estilos de vida.

De manera general, una de las conclusiones a las que han llegado los estudios antes citados (26-41) es que las experiencias previas que tienen las personas con hábitos y estilos de vida saludables como la práctica de ejercicio y sus beneficios, se establecen como factores protectores que están relacionados con la salud, inclusive si existe una mayor autoeficacia percibida previamente, habrá un mayor compromiso dentro de un plan de atención; sin embargo, si existen barreras es fundamental identificarlas, ya que de lo contrario la intervención fracasará.

Cuando las personas disfrutan de la actividad planeada y esta se incrementa de forma paulatina y programada, se predice una mayor autoeficacia. Los profesionales de enfermería pueden evaluar la experiencia previa de autoeficacia y enfatizar en el diseño de programas de intervención, para ayudar a los pacientes con riesgo cardiovascular o enfermedad crónica a ser más participativos en su cuidado. 
Ante este contexto, en el siguiente apartado plantea detalladamente una intervención de enfermería fundamentada en los principales supuestos del MPS, integrando el uso de tecnologías de la información, con la finalidad de dar alternativas a la solución de problemas de salud pública relacionados con EC y FRCV.

\section{El cuidado de enfermería a la persona con Enfermedad Cardiovascular Y Factores de Riesgo Cardiovascular.}

Si bien el cuidado debe ser humanizado y basado en la evidencia, también es fundamental hacer uso de teorías o modelos de enfermería que guíen las intervenciones, tal es el caso del MPS, que como se mencionó anteriormente permite comprender comportamientos humanos relacionados con las enfermedades cardiovasculares y los FRCV, y a su vez, orienta hacia la generación de conductas saludables. No obstante, se requiere de protocolos de intervención que permitan dar solución a los problemas de salud de la población.

\begin{tabular}{|c|c|c|}
\hline & \\
Invitación con tripticos y cartel con leyendas motivadoras \\
Autoeficacia percibida y factores personales \\
Percepción de salud \\
FRCV
\end{tabular}

Figura 2. El cuidado de enfermería relacionado con el MPS de Pender. 
Se propone, con base en la evidencia, que en la práctica de enfermería se desarrollen intervenciones educativas, costo-efectivas, eficientes, viables y tangibles, que impacten en los indicadores de salud sin dejar de considerar las preferencias de los pacientes en las decisiones sobre el cuidado de su salud, esto con el objetivo de que las estrategias contemplen la participación de la población y se acerquen más a la realidad social (fig.2) (42, 43).

La primera condición es que los pacientes participen libre y voluntariamente dentro de una intervención educativa y se proceda a determinar basalmente la autoeficacia percibida, la percepción de su salud, los FRCV, el perfil de estilos de vida saludable y los factores personales; entre otros, para así conocer con exactitud las creencias que tienen las personas sobre sus propias capacidades, y de esa forma organizar y ejecutar los cursos de acción requeridos con respecto al manejo de situaciones futuras donde se logre influir sobre el modo de pensar, sentir, motivar y actuar $(3,4)$.

En un segundo momento, considerando las experiencias previas y la persuasión del paciente, se proporciona un curso de educación para la salud personalizado o grupal con la participación de un "paciente modelo". Esta estrategia pedagógica se ejecutará desde la hospitalización hasta el egreso, dándose seguimiento domiciliario. Este proceso debe contener 3-12 sesiones de 30-60 minutos durante la(s) semana(s) de hospitalización y seguimiento a través un guión o manual para limitar la variabilidad de la información hasta por 3-6 meses (44-46). También es importante desarrollar talleres para la salud relacionados con adherencia a nutrición saludable, actividad física, autocuidado, tratamiento farmacológico, entre otros. En este proceso se deben incluir herramientas didácticas, escritas o electrónicas; tales como folletos o videograbaciones y un plan de cuidados que permita reforzar el proceso enseñanza-aprendizaje. El éxito en el cambio de comportamientos que tienen los pacientes modelos, puede influir en la acogida que otras personas den a la estrategia, sobre todo cuando estas observan que otros similares alcanzan el éxito a través de esfuerzos perseverantes. Esto conduce al fortalecimiento de creencias en relación a que cada uno posee las capacidades necesarias para desarrollar actividades provechosas que puedan ser comparables. Por lo tanto, mientras mayor semejanza observe el paciente en el modelo, más fácil será persuadirlo y tener éxito en el cambio de conducta (V. fig.1).

El tercer punto a realizar es la evaluación del efecto que tiene la intervención sobre las variables o factores medidos basalmente. Finalmente, el seguimiento es fundamental si se pretende mantener el efecto por el mayor tiempo posible y que se traduzca en un cambio de conductas saludables. De acuerdo a la evidencia se propone dar seguimiento por lo menos 2 años a través de la enfermería familiar (47-49), la consultoria de enfermería y/o la visita domiciliaria, haciendo uso de las TIC's. Si se considera que por lo menos $80 \%$ de las personas cuentan con un dispositivo móvil como el celular o la Tablet (50), se puede hacer uso de una aplicación tecnológica que active mensajes persuasivos durante las semanas de seguimiento sobre la conducta a modificar $(51,52)$. La tele-enfermería es otra herramienta que se puede considerar (53), ya que por lo menos el $42 \%$ de las personas tiene un teléfono fijo (47) con 
el cual se puede dar vigilancia y continuidad del cuidado planeado al alta del paciente. El internet, también es una estrategia que puede utilizar el profesional de enfermeria, sobre todo porque el $30.7 \%$ de la población tiene acceso (47); la web puede acercar al personal de salud con el paciente y su familia. El cuidado puede proporcionarse, de forma potencial, con mayor calidad y seguridad, considerando los diferentes software que existen en la web o que se pueden diseñar especialmente para la intervención. Ahora es más fácil tener una videoconferencia, comunicarse a través de la web o asesorar a distancia y observar la clínica del paciente. Las TICś como una estrategia de soporte social, son de fácil acceso y permiten el seguimiento, control, vigilancia, educación, acceso a la asesoría domiciliaria de pacientes con enfermedades crónicas no transmisibles donde se tenga en cuenta a la familia y al cuidador principal. Esta alternativa ha mostrado que optimiza los gastos médicos, disminuye el reingreso hospitalario y mejora la calidad de vida de los pacientes (54-56). Otra variable que es elemental considerar, es el estado psicológico y emocional del paciente, ya que el estado de ánimo positivo fomenta la autoeficacia percibida y desempeña un rol influyente en el funcionamiento de la salud y en el desarrollo de actividades que requieran esfuerzo físico y persistencia. De igual manera se debe valorar el contexto social y económico, ajustando la intervención de acuerdo al contexto de cada paciente.

El cuidado de enfermería a través de intervenciones educativas, independientemente de que haya sido transmitida de manera activa, vicaria, persuasiva o afectivamente, adquiere su importancia mediante el procesamiento cognitivo. El contexto sociocultural y los factores personales, sociales, situacionales, influyen sobre la interpretación de las experiencias relevantes a la eficacia.

\section{Conclusión}

Los modelos y teorías de enfermería no son verdades absolutas, sino maneras de ver un fenómeno. En muchos casos tienen limitaciones o condiciones que deben considerarse antes que el profesional de enfermería los lleve a la práctica clínica para la solución de problemas de salud como las enfermedades cardiovasculares y la presencia de FRCV.

Se ha demostrado que el MPS sirve para identificar conductas, hábitos y estilos de vida y explica las relaciones entre los factores que se cree influyen en las modificaciones de la conducta de salud, el uso de las habilidades y conocimientos de las personas, la adopción de estilos de vida saludables, cambios de comportamiento, y hábitos de actividad física. Así mismo, se ha comprobado que es aplicable en la clínica y en la investigación de enfermería, ya que facilita la elección de un modelo de cuidado y revela posibilidades y limitaciones que pueden enriquecer la práctica profesional.

Las intervenciones de enfermería basadas en la evidencia y los principales supuestos teóricos del MPS pueden contribuir a la solución de los problemas de salud de la población, tanto para disminuir la recaída de la enfermedad, reingresos hospitalarios, complicaciones y la presencia de un reinfarto en el paciente cardiópata, como para controlar y disminuir los efectos de los FRCV. 


\section{Conflicto de interés}

Los autores declaran no tener conflictos de interés.

\section{Financiación}

Ninguno

\section{Referencias}

1. World Health Organization. Global status report on noncommunicable diseases 2014. Switzerland: OMS; 2014.

2. Marriner TA, Raile AM, Modelos y teorías de enfermería. 6ta ed. España: Elsevier Science; 2007.

3. Bandura A. Autoeficacia: cómo afrontamos los cambios de la sociedad actual. España: Desclée de Brouwer; 1999.

4. Bandura A. Self-efficacy: Toward a unifyng theory of behavioral change. Psychology review. 1977; 84(2): 191-215.

5. Ariztizabal HGP, Marlene Blanco BD, Sánchez RA, Ostiguín MRM. E1 modelo de promoción de la salud de Nola Pender. Una reflexión en tono a su comprensión. Enferm Universitaria-Mex. 2011; 8(8):16-23.

6. Mellado-Sampedro MT, Pérez-Noriega E, Árcega-Domínguez A, SorianoSotomayor MA, Arrioja-Morales G. Estilo de vida: Precursor de factores de riesgo cardiovascular en adultos sanos. Rev Mex Enferm Cardiol. 2011; 19(2): 56-61.

7. Galicia ARM, Nájera GG, Morales NA, Villegas VMS. Promoción de Conductas Saludables en Personas con Diabetes Mellitus tipo 2. Desarrollo Cientif Enferm. Sept. 2009; 17(8): 355-7.

8. Eshah NF, Bond AE, Froelicher ES. The effects of a cardiovascular disease prevention program on knowledge and adoption of a heart healthy lifestyle in Jordanian working adults. Eur J Cardiovasc Nurs. [Internet] 2010 Mar 17 [Consultado en nov 05 de 2014]; Dec; 9(4): 24453. Disponible en: http://doi: 10.1016/j.ejcnurse.2010.02.002

9. Srof BJ, Velsor-Friedrich B. Health promotion in adolescents: a review of Pender's health promotion model. Nurs Sci Q. 2006 Oct; 19(4): 366-73.

10. Harooni J, Hassanzadeh A, Mostafavi F. Influencing factors on health promoting behavior among the elderly living in the community. J Educ Health Promot. eCollection. [Internet] 2014 May 5 [Consultado en nov 6 de 2014] 3:40.Disponible en: http://doi: 10.4103/2277-9531.131921

11. Baker JR. Cultural influences on health-promoting behaviors of older African-American women. J Natl Black Nurses Assoc. 2011 Dec; 22(2): 53-8.

12. Backes DS, Backes MTS, Rangel RF, Erdmann AL, Büscher A. Significado de viver saudável para usuários, profissionais e gestores da Saúde. Rev Bras Enferm, Brasilia 2011 nov-dez; 64(6): 1094-9.

13. Cho SH, Choi M, Lee J, Cho H. Relationship between Expectations Regarding Aging and Physical Activity among Middle Aged Adults in Urban Areas: Based on the Pender's Health Promotion Model. J Korean Acad Nurs. [Internet] 2015 Feb 27 [Consultado en jul 10 2015]; 45(1): 14-24. Disponible en: http://doi: 10.4040/jkan.2015.45.1.14. 
14. Monteiro FPM, Oliveira CJ, Vitor AF, Araújo TL, Ximenes LB. Avaliação do estado nutricional de crianças com cardiopatia congênita sob a ótica de Pender. Rev. enferm. UERJ, Rio de Janeiro, 2009 out/dez; 17(4): 581-8.

15. Ho AY, Berggren I, Dahlborg-Lyckhage E. Diabetes empowerment related to Pender's Health Promotion Model: a meta-synthesis. Nurs Health Sci. [Internet] 2010 Jun [Consultado en oct 23 de 2014]; 12(2): 259-67. Disponible en: http://doi: 10.1111/j.1442-2018.2010.00517.x.

16. Restrepo HE, Málaga H. Promoción de la salud: cómo construir vida saludable. Colombia: Panamericana; 2001.

17. Silva ACS, Santos I. Promoção do autocuidado de idosos para o enve1hecer saudável: aplicação da teoria de Nola Pender. Texto Contexto Enferm, Florianópolis, 2010 Out-Dez; 19(4): 745-53.

18. Chase JA, Conn VS. Meta-analysis of fitness outcomes from motivational physical activity interventions. Nurs Res. [Internet] 2013 SepOct [Consultado en nov 11 de 2014]; 62(5): 294-304. Disponible en: http://doi: 10.1097/NNR.0b013e3182a0395c.

19. Kamran A, Azadbakht L, Sharifirad G, Mahaki B, Mohebi S. The relationship between blood pressure and the structures of Pender's health promotion model in rural hypertensive patients. J Educ Health Promot. eCollection [Internet] 2015 Mar 27[Consultado en jul 10 de 2015]; 4: 29. Disponible en: http://doi: 10.4103/2277-9531.154124.

20. Dehdari T, Rahimi T, Aryaeian N, Gohari MR, Esfeh JM. Developing and testing a measurement tool for assessing predictors of breakfast consumption based on a health promotion model. J Nutr Educ Behav. [Internet] Mar 2014 [Consultado en jul 10 de 2015]; 46(4): 250-8. Disponible en: http://doi: 10.1016/j.jneb.2013.12.007.

21. Mohamadian H, Eftekhar H, Rahimi A, Mohamad HT, Shojaiezade D, Montazeri A. Predicting health-related quality of life by using a health promotion model among Iranian adolescent girls: A structural equation modeling approach. Nurs Health Sci. Epub [Internet] 2011 May [Consultado en nov 11 de 2014]; 13(2): 141-8.Disponible en: http:// doi: 10.1111/j.1442-2018.2011.00591.x.

22. Santos I, Soares CS, Berardinelli LMM. Promovendo o autocuidado de clientes com obesidade e coronariopatia: aplicação do diagrama de Pender. Rev. enferm. UERJ, Rio de Janeiro, 2013 jul/set; 21(3): 301-6.

23. Kamran A, Azadbakht L, Mahaki B, Sharifirad G. Psychometric testing of an instrument designed to measure nutritional perceptions of hypertensive patients. J Educ Health Promot. eCollection. [Internet] Aug 2014 [Consultado en nov 11 de 2014]; 28 (3): 91. Disponible en: http://doi: 10.4103/2277-9531.139662. 2014.

24. Bezares-Sarmiento VR, Bacardi-Gascón M, Márquez-Rosa S, MolineroGonzález O, Estrada-Grimaldo M, Jiménez-Cruz M. Efficacy of social support on metabolic syndrome among low-income rural women in Chiapas, México. Nutr Hosp. 2013; 28: 1195-200.

25. Medina LOM, Dáz PAD, Barrientos GE, Peña MDE. Percepción de salud y su efecto en pacientes con diabetes. Av. enferm. 2009; 27(2): 13-8. 
26. Triviño Z, stiepovich J, Merino JM. Factores predictores de conductas promotoras de salud en mujeres peri- post-menopáusicas de Cali, Colombia. Colomb Med 2007; 38(4): 395-407.

27. Victor JF, Lopes MVO, Ximenes LB. Análise do diagrama do modelo de promoção da saúde de Nola J. Pender. Acta Paul Enferm. 2005; 18(3): 235-40.

28. Triviño-Vargas $Z$ G. Conductas promotoras de salud en estudiantes de una institución de educación superior. Aquichan. 2012; 12(3): 275-85.

29. Monteiro FPM, Oliveira CJ, Vitor AF, Araújo TL, Ximenes LB. Avaliação do estado nutricional de crianças com cardiopatia congênita sob a ótica DE Pender. Rev. enferm. UERJ. Oct-dic 2009; 17(4): 581-8.

30. Santos SSC, Barlem ELD, Silva BT, Cestari ME, Lunardi VL. Health promotion for the elderly: gerontogeriatric nursing Commitment. Acta Paul Enferm. 2008; 21(4): 649-53.

31. Silva ACS, Santos I. Promoção do autocuidado de idosos para o envelhecer saudável: aplicação da teoria de Nola Pender. Texto Contexto Enferm, Florianópolis, 2010; 19(4): 745-53.

32. Guedes NG, Moreira RP, Cavalcante TF, Araujo TL, Ximenes LB. Students' physical activity: an analysis according to Pender's health promotion model. Rev Esc Enferm USP. 2009; 43(4): 773-9.

33. Shin Y, Pender NJ, Yun S. Using methodological triangulation for cultural verification of commitment to a plan for exercise scale among Korean adults with chronic diseases. Res Nurs Health. Agost 2003; 26(4): 312-21.

34. Shin Y, Yun S, Pender NJ, Jang H. Test of the Health Promotion Model as a Causal Model of Commitment to a Plan for Exercise Among Korean Adults With Chronic Disease. Res Nurs Health. 2005 Apr; 28(2): 117-25.

35. Shin $\mathrm{YH}^{1}$, Hur HK, Pender NJ, Jang HJ, Kim MS. Exercise self-efficacy, exercise benefits and barriers, and commitment to a plan for exercise among Korean women with osteoporosis and osteoarthritis. Int J Nurs Stud. 2006; 43(1): 3-10.

36. Pender NJ, Bar-Or O, Wilk B, Mitchell S. Self-efficacy and perceived exertion of girls during exercise. Nurs Res. Mar-Abr 2002; 51(2): 86-91.

37. Robbins LB, Pis MB, Pender NJ, Kazanis AS. Physical activity selfdefinition among adolescents. Res Theory Nurs Pract. 2004 Winter; 18(4): 317-30.

38. Robbins LB, Pis MB, Pender NJ, Kazanis AS. Exercise self-efficacy, enjoyment, and feeling states among adolescents. West J Nurs Res. Nov 2004; 26(7): 699-721.

39. Robbins LB, Pender NJ, Kazanis AS. Barriers to physical activity perceived by adolescent girls. J Midwifery Womens Health. May-jun 2003; 48(3): 206-12.

40. Padden DL, Connors RA, Posey SM, Ricciardi R, Agazio JG. Factors Influencing a Health Promoting Lifestyle in Spouses of Active Duty Military. Health Care Women Int. Epub [Internet] 2013 Mar 26 [Consultado en nov 11 de 2014];34(8):674-93. doi: 10.1080/07399332.2012.736572. 
41. Carreño J, Vyhmeister G, Grau L, Ivanovic D. A health promotion programme in Adventist and non-Adventist women based on Pender's model: a pilot study. Public Health. 2006 Apr;120(4):346-55. Epub $2006 \mathrm{Feb} 14$.

42. Horta NC, de Sena RR, Silva MEO, de Oliveira SR, Rezende VA. A prática das equipes de saúde da família: desafios para a promoção de saúde. Rev Bras Enferm, Brasília 2009 jul-ago; 62(4): 524-9.

43. Pereira IC, Oliveira MAC. O trabalho do agente comunitário na promoção da saúde: revisão integrativa da literatura. Rev Bras Enferm, Brasilia 2013 mai-jun; 66(3): 412-9.

44. Hudon C, Fortin M, SoubhiH.Single risk factor interventions to promote physical activity among patients with chronic diseases. Systematic review. Can Fam Physician 2008; 54: 1130-7.

45. Seitz P, Rosemann T, Gensichen J, Huber CA. Interventions in primary care to improve cardiovascular risk factors and glycatedhaemoglobin (HbA1c) levels in patients with diabetes: a systematic review. Diabetes, Obesity and Metabolism. 2011;13: 479-89.

46. Groeneveld IF, Proper KI, van der Beek AJ, Hildebrandt VH, van Mechelen W. Lifestyle-focused interventions at the workplace to reduce the risk of cardiovascular disease-a systematic review.Scand $\mathrm{J}$ WorkEnvironHealth. [Internet] 2010 [Consultado en] nov $11 \mathrm{de}$ 2014;36(3):202-215 Disponible en: http://doi:10.5271/sjweh.2891.

47. Gurgel MGI, Alves MDS, Moura ERF, Pinheiro PNC, Rêgo RMV, Passos MLL. Promoção da saúde no contexto da estratégia saúde da família: concepções e práticas da enfermeira. Esc Anna Nery (impr.)2011 jul-set; 15 (3):610-5.

48. Mena-Gómez I, Cadena-Estrada JC, Troncoso-Pérez D. Intervención de enfermería en una familia mexicana con factores de riesgo para enfermedades crónico-degenerativa. Enfermería Universitaria. 2014; 11(2): 67-72.

49. Paz EPA, Souza MHN, Guimarães RM, Pavani GF, Correa HFS, Carvalho PM et al. Estilos de vida de pacientes hipertensos atendidos com a Estratégia de Saúde Familiar. Invest Educ Enferm. 2011;29(3): 467-476.

50. Instituto Nacional de Estadística y Geografía. Estadisticas sobre disponibilidad y uso de tecnología de información y comunicaciones en los hogares 2012. México: INEGI [Internet]2013 [citado el 12 ene 2015] Disponible en:http://www.inegi.org.mx/prod_serv/contenidos/espanol/bvinegi/productos/encuestas/especiales/endutih/endutih2012.pdf

51. Márquez CE, Figuera WM, Gil GV, Ylla-Catalá A, Figueras M, Balaña M, Naval J. Eficacia de una intervención informativa a hipertensos mediante mensajes de alerta en el teléfono móvil (HTA-ALERT). Aten Primaria 2004; 34(8): 399-407.

52. Piette JD, Lange I, Issel M, Campos S, Bustamante C, Sapag J, Poblete F, Tugwell P, O'Connor AM. Use of telephone care in a cardiovascular disease management programme for type 2 diabetes patients in Santiago, Chile. Chronic Illness [Internet] 2006 [Consultado en nov 11 de 2014l:2, 87-96. Disponible en: http://doi: 10.1179/174592006X110969 
53. Chaves EC, Oyama SMR. Modelo de intervenção telefônica na sensibilização de mudança do padrão alimentar. RBPS, Fortaleza. abr./ jun 2010; 23(2): 136-43.

54. Hamel LM, Robbins LB, Wilbur J. Computer- and web-based interventions to increase preadolescent and adolescent physical activity: a systematic review. J Adv Nurs. 2011 Feb;67(2):251-68. doi: 10.1111/j.13652648.2010.05493.x. Epub 2010 Dec 28.

55. Campos de Aldana MS, Moya-Plata D, Mendoza-Matajira JD, DuranNiño EY. Las enfermedades crónicas no transmisibles y el uso de tecnologías de información y comunicación: revisión sistemática. Rev Cuid. 2014; 5(1): 661-9.

56. Bradbury-Golas K. Health promotion and prevention strategies. Nurs Clin North Am. [Internet] 2013 [Consultado en nov 11 de 2014]; Sep; 48(3): 469-83. Disponible en: http://doi: 10.1016/j.cnur.2013.04.003. Epub 2013 Jul 22. 